\title{
Desigualdades na mortalidade por doenças crônicas entre idosos e sua associação com indicadores socioeconômicos no Brasil
}

\author{
Davi Barroso Alves", Maria Tereza Serrano Barbosa"*
}

\section{Resumo}

O processo de envelhecimento populacional no Brasil, decorrente da queda nas taxas de natalidade e mortalidade infantil e do aumento na expectativa de vida, acarreta mudanças na mortalidade da população, que deixa de ser caracterizada por processos tipicamente agudos, passando a ser caracterizada principalmente por processos crônicos, que geram anos de utilização do sistema de saúde. Essa mudança representa um problema para o setor saúde, que não está totalmente preparado para acolher essa população e tratar de forma adequada seus problemas crônicos de saúde. Nesse sentido, a compreensão das desigualdades na mortalidade por doenças crônicas entre idosos e sua associação com indicadores socioeconômicos no país pode ser de grande ajuda na promoção de saúde para essa população. Assim, utilizou-se no presente estudo a análise estatística de dados epidemiológicos, populacionais e socioeconômicos com a finalidade de analisar as desigualdades e contribuir na discussão de ações eficientes na manutenção e promoção da saúde da população idosa. Percebeu-se que as regiões Norte e Nordeste apresentam piores indicadores socioeconômicos e menores taxas de mortalidade por doenças crônicas, além de estarem em um estágio inicial ou intermediário de envelhecimento populacional. Em contrapartida, as UF que compõem as regiões Sul, Sudeste e Centro-Oeste apresentam um estágio mais avançado de envelhecimento, melhores indicadores socioeconômicos e maiores taxas de mortalidade por doenças crônicas. Percebe-se, assim, a necessidade de uma abordagem diferenciada para o controle das doenças crônicas no Brasil e para a promoção da saúde da população idosa.

Palavras-chave: Envelhecimento. Desigualdade. Doenças crônicas. Saúde do idoso.

" Acadêmico do curso de Enfermagem da Universidade Federal do Estado do Rio de Janeiro. Endereço para correspondência: Rua Ilka Brasil Barbosa, 120, apto 303, bairro Fonseca, CEP: 24130-026, Niterói - RJ. E-mail: davialves01@hotmail.com.

* Doutora em Saúde Coletiva pela Universidade do Estado do Rio de Janeiro. Professora do Departamento de Matemática e Estatística da Universidade Federal do Estado do Rio de Janeiro.

$\hookrightarrow$ Recebido em setembro de 2008 - Avaliado em agosto de 2009.

$\hookrightarrow$ doi: $10.5335 /$ rbceh.2010.003 


\section{Introdução}

O Brasil experimenta, desde a década de 1940, queda nas taxas de mortalidade, principalmente na mortalidade infantil. Em virtude da manutenção da taxa de natalidade, a população brasileira aumentou de 41 para 93 milhões de pessoas entre 1940 e 1970 (CHAIMOWICZ, 1997) e, a partir de 1960, com o início da queda da taxa de natalidade nos estados mais desenvolvidos do país, iniciou-se o processo de transição demográfica da população brasileira em direção ao envelhecimento. Tal processo se deu de forma heterogênea, havendo diferenças substanciais entre a estrutura etária das diversas UF.

Até então, esse processo era preocupação exclusiva das nações mais desenvolvidas do globo, que apresentavam baixa taxa de mortalidade, tanto pelo elevado desenvolvimento médico sanitário quanto pela melhor qualidade de vida da população; baixa taxa de natalidade, fruto do planejamento familiar associado ao desenvolvimento de métodos anticoncepcionais mais eficazes, e alta expectativa de vida ao nascer, que, associados, culminavam no processo de envelhecimento populacional.

Nesses países, esse processo foi acompanhado pela ampliação da cobertura dos sistemas de proteção social e melhorias das condições de habitação, alimentação, trabalho e do saneamento básico, que permitiram a manutenção de uma boa qualidade de vida para toda a população (CHAIMOWICZ, 1997; KALACHE; VERAS; RAMOS, 1987). No Brasil, a queda dessas taxas ocorreu, sobretudo, pela ação médico-sanitária do Estado e não foi acompanhada por transformações estruturais que pudessem ser traduzidas em melhoria na qualidade de vida dos brasileiros, o que gera um grave problema de ordem social e econômica na assistência a essa população idosa em expansão.

Biologicamente, o envelhecer é resultado do desgaste natural das estruturas orgânicas, prevalecendo com o tempo os processos degenerativos (JARDIM; MEDEIROS; BRITO, 2006), caracterizados principalmente pelos problemas crônicos, que demandam anos de utilização dos serviços de saúde. O processo de envelhecimento populacional provoca alteração no perfil epidemiológico da população. Segundo Chaimowicz (1997), "modifica-se o perfil de saúde da população; ao invés de processos agudos que 'se resolvem' rapidamente através da cura ou do óbito, tornam-se predominantes as doenças crônicas e suas complicações, que implicam em décadas de utilização dos serviços de saúde".

Dados do Sistema de Informações Hospitalares do SUS (SIH/SUS) mostram que, no período de janeiro a outubro de 2007, 4,87 de cada cem adultos (indivíduos de 15 a 59 anos) foram internados, ao passo que 11,95 de cada cem idosos (indivíduos com sessenta anos ou mais) foram internados no mesmo período no país. Em alguns estados, como o Paraná, essa diferença é ainda maior, sendo de 5,41 a cada cem adultos para 17,24 a cada cem idosos internados. Além disso, o número de internações de pessoas com sessenta anos ou mais de idade subiu de 1.285.163 em 2002 
para 1.907.470 em 2007, representando um aumento de mais de $48 \%$, enquanto entre adultos houve uma ligeira queda, de $3,3 \%$, reduzindo-se de $7.084 .449 \mathrm{em}$ 2002 para 6.858 .833 em 2007.

O modelo assistencial atual no Brasil está centrado no tratamento de casos agudos, caracterizando-se pela procura de tratamento de sintomas iguais repetidas vezes e sendo pouco eficiente no tratamento de situações crônicas de saúde. (TRENTINI et al., 2005). Um modelo de atendimento à saúde nunca poderá deixar de ter tratamento eficaz para as condições agudas, afinal, as doenças crônicas passam por fases agudas, e um tratamento eficiente destas pode ser uma forma eficiente de se prevenirem estados crônicos. Porém, tão importante quanto um bom tratamento de estágios agudos é a prevenção dessas doenças por meio de ações centradas na educação em saúde e em ações específicas, já que os avanços no atendimento a doenças crônicas permitem uma sobrevida maior aos acometidos por estas, apesar de nem sempre significar melhor qualidade de vida.

Nesse contexto, observa-se que a doença não se distribui de forma homogênea no país, pois atinge grupos populacionais em momentos, formas e intensidades distintos (CORDEIRO et al., 1999), e cada um desses grupos possui uma condição própria de existência, caracterizada pela desigualdade social, cultural e econômica. Nessa mesma visão, Duarte et al. (2002) ressaltam que são vários os aspectos que determinam essas diferenças, tais como as desigualdades na distribuição dos fatores de exposição às doenças e o acesso aos bens e serviços de saúde, a fragilidade das estruturas sociais de apoio à saúde e o insuficiente investimento em políticas sociais, de forma especial em sociedades com alta concentração de renda e nível de coesão social. Desse modo, a compreensão das diferenças no processo de envelhecimento nas diversas regiões do país, levando em consideração os diversos fatores sociais, econômicos e culturais que influenciam nesse processo, pode ajudar na promoção de saúde, em seu sentido mais amplo, da população idosa do país.

Estudos como o de Chiesa, Westphal e Akerman (2008), Cordeiro et al. (1999) e Ishitani et al. (2006) evidenciam a importância de se aplicarem métodos quantitativos que permitam relacionar indicadores socioeconômicos com coeficientes de mortalidade, com o objetivo de apontar desigualdades em saúde no Brasil.

Fica evidente que num processo de envelhecimento populacional é necessário buscar compreender a desigualdade na mortalidade entre idosos por meio de estudos que levem em consideração aspectos que são pertinentes a esta população.

O presente artigo procura analisar as desigualdades na mortalidade por doenças crônicas entre idosos nas unidades da federação do Brasil, relacionando-as com as características socioeconômicas das mesmas, visando contribuir na discussão a respeito de ações que sejam eficientes na manutenção e na promoção da saúde da população idosa. 


\section{Metodologia}

Para o desenvolvimento do presente artigo realizou-se um estudo analítico do tipo ecológico, tendo como unidades de observação as 27 UF do Brasil, utilizando-se dados retirados do Sistema de Informações sobre Mortalidade (SIM), do Sistema de Informações de Nascidos Vivos (Sinasc), das estimativas preliminares dos totais populacionais segundo o Censo 2000 e Contagem Populacional de 1996 e de estudos sobre o Índice de Desenvolvimento Humano nas UF do Brasil realizadas pelo Programa das Nações Unidas para o Desenvolvimento (PNUD).

As variáveis utilizadas foram os coeficientes de mortalidade pelas principais doenças crônicas e indicadores socioeconômicos, conforme apresentados no Quadro 1:

Quadro 1 - Resumo dos coeficientes.

\begin{tabular}{|c|c|c|}
\hline Coeficiente & Descrição & Dados utilizados \\
\hline $\begin{array}{l}\text { Coeficiente de } \\
\text { mortalidade por } \\
\text { neoplasias entre } \\
\text { idosos em } 2005 \\
\text { (CMN) (CID-10: II) }\end{array}$ & $\begin{array}{l}\text { Número de óbitos por neoplasias } \\
\text { e tumores entre idosos em uma } \\
\text { população a cada dez mil habitantes } \\
\text { nesta faixa etária }\end{array}$ & $\begin{array}{l}\text { - Número de óbitos por neoplasias e } \\
\text { tumores entre idosos } \\
\text { - População da área no período, } \\
\text { estratificada por idade }\end{array}$ \\
\hline $\begin{array}{l}\text { Coeficiente de } \\
\text { mortalidade por } \\
\text { doença do aparelho } \\
\text { respiratório entre } \\
\text { idosos em } 2005 \\
(\text { CMR) (CID-10: X) }\end{array}$ & $\begin{array}{l}\text { Número de óbitos por doenças do } \\
\text { aparelho respiratório entre idosos } \\
\text { em uma população a cada dez mil } \\
\text { habitantes nesta faixa etária }\end{array}$ & $\begin{array}{l}\text { - Número de óbitos por doenças do } \\
\text { aparelho respiratório entre idosos } \\
\text { - População da área no período, } \\
\text { estratificada por idade }\end{array}$ \\
\hline $\begin{array}{l}\text { Coeficiente de } \\
\text { mortalidade por } \\
\text { doença do aparelho } \\
\text { circulatório entre } \\
\text { idosos em } 2005 \\
(\text { CMC) (CID-10: IX) }\end{array}$ & $\begin{array}{l}\text { Número de óbitos por doenças do } \\
\text { aparelho circulatório entre idosos } \\
\text { em uma população a cada dez mil } \\
\text { habitantes nesta faixa etária }\end{array}$ & $\begin{array}{l}\text { - Número de óbitos por doenças do } \\
\text { aparelho circulatório entre idosos } \\
\text { - População da área no período, } \\
\text { estratificado por idade }\end{array}$ \\
\hline $\begin{array}{l}\text { Índice de } \\
\text { desenvolvimento } \\
\text { humano em } 2000 \\
\text { (IDH) }\end{array}$ & $\begin{array}{l}\text { Índice calculado com base em } \\
\text { informações de educação, saúde e } \\
\text { renda de uma determinada população }\end{array}$ & $\begin{array}{c}\text { - Índice retirado do site do Programa das } \\
\text { Nações Unidas pelo Desenvolvimento }\end{array}$ \\
\hline $\begin{array}{l}\text { Taxa de mortalidade } \\
\text { infantil em } 2005 \text { (TMI) }\end{array}$ & $\begin{array}{l}\text { Número de óbitos de indivíduos com } \\
\text { menos de um ano de idade para cada } \\
\text { mil nascidos vivos }\end{array}$ & $\begin{array}{l}\text { - Número de óbitos entre indivíduos com } \\
\text { menos de um ano de idade } \\
\text { - Número de nascidos vivos }\end{array}$ \\
\hline $\begin{array}{l}\text { Índice de } \\
\text { envelhecimento } \\
\text { populacional em } 2005 \\
\text { (IEP) }\end{array}$ & $\begin{array}{l}\text { Número de indivíduos com } 64 \text { anos de } \\
\text { idade ou mais de um ano de idade para } \\
\text { cada cem indivíduos com menos de } 15 \\
\text { anos de idade }\end{array}$ & $\begin{array}{l}\text { - Número de indivíduos com } 64 \text { anos ou } \\
\text { mais de idade } \\
\text { - Número de indivíduos com menos de } 15 \\
\text { anos de idade }\end{array}$ \\
\hline $\begin{array}{l}\text { Mortalidade } \\
\text { proporcional por } \\
\text { causas mal definidas } \\
\text { em } 2005 \text { (MCMD) } \\
\text { (CID-10: XVIII) }\end{array}$ & $\begin{array}{l}\text { Número de óbitos por Sintomas, } \\
\text { sinais e achados anormais de } \\
\text { exames clínicos e de laboratório, não } \\
\text { classificados em outra parte dividido } \\
\text { pelo total de óbitos }\end{array}$ & $\begin{array}{l}\text { - Número de óbitos por sintomas, sinais e } \\
\text { achados anormais de exames clínicos e } \\
\text { de laboratório, não classificados em outra } \\
\text { parte } \\
\text { - Número total de óbitos da área no período }\end{array}$ \\
\hline
\end{tabular}


A mortalidade proporcional por causas mal definidas foi utilizada para analisar e discutir a diferença na qualidade dos dados de mortalidade nas diversas UF.

Com o objetivo de obter o perfil epidemiológico e social das unidades da federação em relação às distribuições das taxas de mortalidade, do IDH e IEP, realizou-se na primeira etapa uma análise exploratória, por meio de medidas de posição (média e mediana), medidas de dispersão (desvio padrão [DP] e quartis), gráficos (histograma e diagrama de caixas) e mapas. Na segunda etapa, analisou-se o comportamento conjunto das taxas e indicadores por meio das medidas de correlação de Pearson.
Os dados foram organizados e analisados com o auxílio dos softwares Excel (Microsoft (C), R (The R Foundation For Statistical Computing Copyright (C), 2007) e TABWin (Datasus - Departamento de Informática do SUS (C).

\section{Resultados}

A Tabela 1 mostra os resultados encontrados por região. Pode-se perceber que os estados que compõem as regiões Norte e Nordeste apresentam os menores valores das taxas de mortalidade por doenças crônicas, bem como os piores indicadores socioeconômicos.

Tabela 1 - Resultados encontrados no Brasil e por região.

\begin{tabular}{lcccccc}
\hline \multicolumn{1}{c}{ Região } & $\begin{array}{c}\mathrm{CMN} \\
\mathrm{DP} \pm 15,8\end{array}$ & $\begin{array}{c}\mathrm{CMR} \\
\mathrm{DP} \pm 10,1\end{array}$ & $\begin{array}{c}\mathrm{CMC} \\
\mathrm{DP} \pm 26,2\end{array}$ & $\begin{array}{c}\mathrm{TMI} \\
\mathrm{DP} \pm 2,7\end{array}$ & $\begin{array}{c}\text { IEP } \\
\mathrm{DP} \pm 5,4\end{array}$ & $\begin{array}{c}\text { IDH médio } \\
\mathrm{DP} \pm 0,08\end{array}$ \\
\hline Brasil & 60,6 & 47,8 & 162,0 & 17,0 & 19,5 & 0,77 \\
Norte & 40,2 & 39,3 & 117,3 & 19,6 & 9,6 & 0,73 \\
Nordeste & 41,0 & 31,5 & 135,9 & 20,4 & 17,6 & 0,60 \\
Sudeste & 67,4 & 54,3 & 173,6 & 14,8 & 23,6 & 0,79 \\
Sul & 81,3 & 58,2 & 181,6 & 13,8 & 22,3 & 0,81 \\
Centro-Oeste & 60,4 & 52,9 & 179,8 & 16,3 & 13,9 & 0,79 \\
\hline
\end{tabular}

O coeficiente de mortalidade por neoplasias entre indivíduos com sessenta ou mais anos de idade foi de 60,6 óbitos para dez mil habitantes nessa faixa etária em 2005 , tendo como valor máximo 89,4 , no Rio Grande do Sul, e mínimo de 26,2, no Maranhão. De maneira geral, as UF com os maiores valores para este coeficiente foram as que compõem as regiões Sul e Sudeste do país, com uma média de 71 óbitos para cada dez mil idosos. As UF que compõem as regiões com menores valores de $\mathrm{CMN}$ apresentaram maiores variações, como no Nordeste, onde variou de 26,2, no Maranhão, a 51,6, em Pernambuco.

O coeficiente de mortalidade por doenças do aparelho circulatório entre idosos obteve valores elevados nas UF do Brasil, representando a causa de óbito com maior peso entre as doenças crônicas analisadas. O CMC do Brasil em 2005 foi de 138,2 óbitos para cada dez mil habitantes com sessenta ou mais anos de idade, sendo o mínimo de 71,4, referente ao Amapá, e máximo de 169,6 no Paraná. 
A região Nordeste apresentou as maiores variações desta taxa, entre Bahia $(88,9)$ e Pernambuco $(159,1)$.

A correlação do CMC com os outros coeficientes de mortalidade analisados foi alta, $60 \%$ com o CMN e $61,8 \%$ com o CMR, indicando que as populações com alta mortalidade por algum processo crônico apresentam também altos valores de mortalidade para os outros processos crônicos.

O coeficiente de mortalidade por doenças do aparelho respiratório entre idosos foi o menor entre os coeficientes de mortalidade analisados, apresentando também o comportamento menos desigual quando analisado em termos regionais. O CMR do país em 2005 foi de 47,8 óbitos para cada dez mil idosos, tendo máximo de 62,7 no Rio Grande do Sul e mínimo de 21,4 no Maranhão. A região Norte apresentou a maior variação deste coeficiente, com uma diferença de 31 óbitos para cada dez mil habitantes nesta faixa etária entre o máximo (Acre com 58,4) e o mínimo (Amapá, com 26,8). A correlação entre o coeficiente de mortalidade por doenças do aparelho respiratório e o coeficiente de mortalidade por neoplasias foi de $82,8 \%$, evidenciando que as UF com valores elevados de CMR possuem também valores elevados do CMN, como mostra a Figura 1.

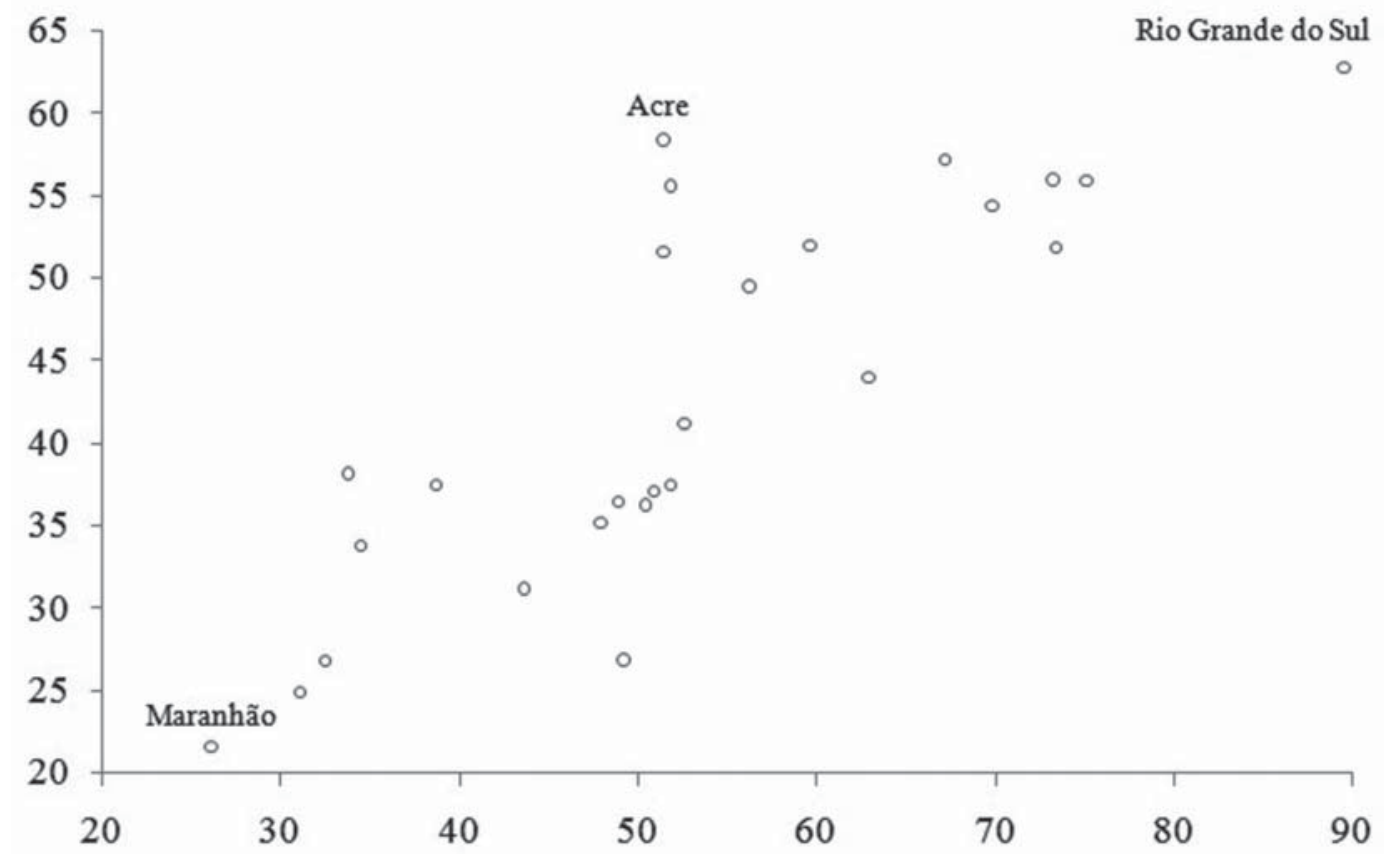

Figura 1 - Correlação entre coeficiente de mortalidade por neoplasias e o coeficiente de mortalidade por doença do aparelho respiratório. 
O índice de desenvolvimento humano (IDH) variou de 0,55 em Alagoas a 0,84 no Distrito Federal. Considerandose que a Organização das Nações Unidas (ONU) preconiza valores abaixo de 0,49 como de baixo desenvolvimento, todas as UF do Brasil apresentam desenvolvimento entre médio (de 0,5 a 0,79 ) e bom ( 0,8 ou mais). Apenas Rio de Janeiro $(0,81)$, São Paulo $(0,82)$, Santa Catarina $(0,82)$, Rio Grande do $\operatorname{Sul}(0,81)$ e Distrito Federal $(0,84)$ possuem IDH classificado como bom. Entre as UF que compõem as regiões Norte e Nordeste, a que possui o maior IDH é o Amapá, com 0,75 , valor que não supera o menor IDH entre as UF que compõem as regiões Sul, Sudeste e Centro-Oeste, que foi de 0,77, no Espírito Santo.

A correlação do IDH foi positiva e alta com o CMN (70\%) e com o CMR $(70,8 \%)$, mostrando que as populações com altos valores de IDH possuem também altos valores de CMN e CMR. Entre o Índice de Desenvolvimento Humano e a taxa de mortalidade infantil a relação foi negativa, $-71,4 \%$, valor já esperado pelo fato de a saúde, no cálculo do IDH, ser representada, entre outros fatores, pela esperança de vida ao nascer, que está diretamente relacionada à queda na taxa de mortalidade infantil.

A taxa de mortalidade infantil no país em 2005 foi de 17,0 óbitos para mil nascidos vivos, com máximo de 24,2 em Alagoas e mínimo de 12,6 em Santa Catarina. Essa variável se comportou de forma muito desigual no país, tendo valores variando muito entre UF que compõem a mesma região, como é o caso da região Centro-Oeste, que teve mínimo de 13,6 no Distrito Federal e máximo de 19,3 no Mato Grosso do Sul, diferença maior que a encontrada entre as UF que compõem a região Norte, que apresentou mínimo de 16,9, no Tocantins, e máximo de 20,9, no Acre.

A TMI apresentou correlação negativa com todas as variáveis estudadas, sendo de $-68,9 \%$ com o CMN, $-39,4 \%$ com o CMC e $-53,9 \%$ com CMR, indicando que as UF com maiores valores de TMI apresentam menores coeficientes de mortalidade por processos crônicos. Entre a TMI e o índice de envelhecimento populacional a correlação foi de $-45,7 \%$, mostrando que o maior envelhecimento populacional possui, além de outros fatores, associação com a queda da taxa de mortalidade infantil.

A Figura 2 mostra a distribuição do CMN, do CMR, do TMI e do IDH no Brasil, por região. Pode-se observar que as regiões Sudeste (SE), Sul (S) e Centro-Oeste (CO) apresentam os maiores valores de CMN e CMR, bem como os maiores valores de TMI e IDH, ao passo que as regiões Norte $(\mathrm{N})$ e Nordeste (NE) apresentam os menores valores das taxas de mortalidade e do IDH e TMI. 
CMN por região

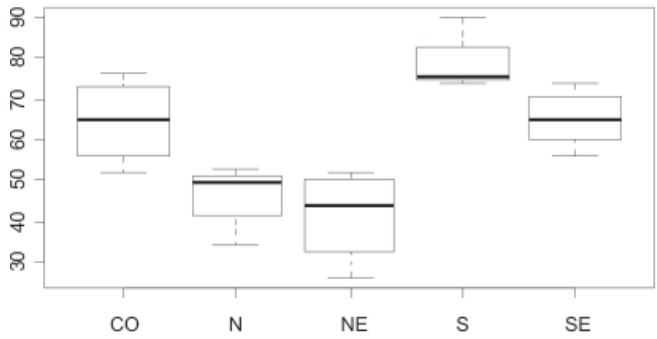

TMI por região

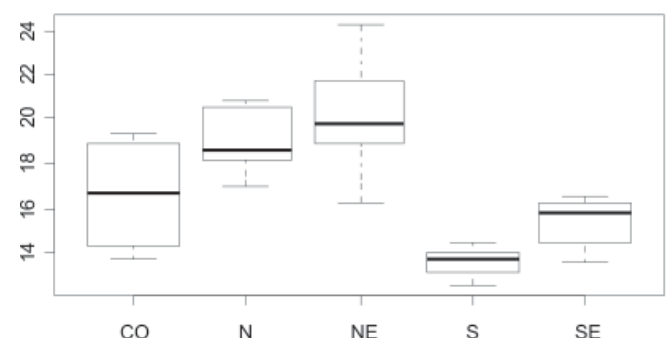

CMR por região

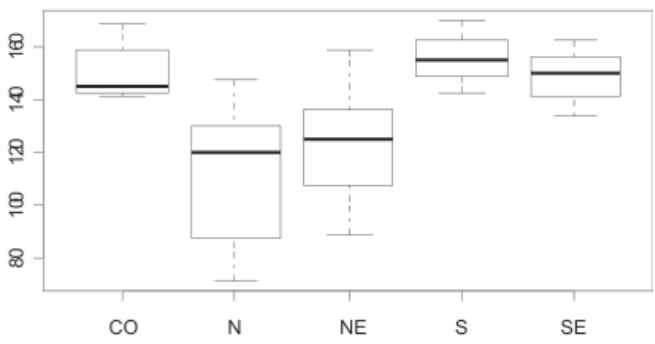

IDH por região

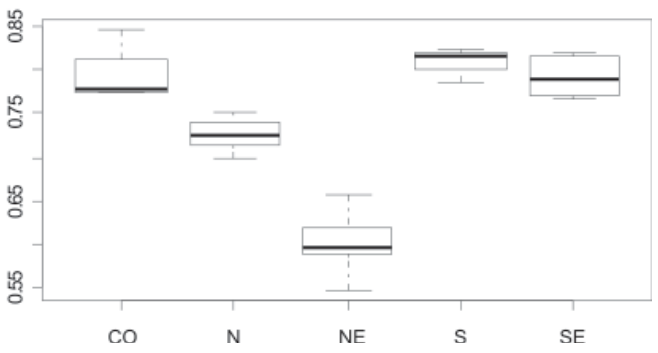

Figura 2 - Coeficiente de mortalidade por neoplasias, coeficiente de mortalidade por doença do aparelho respiratório, taxa de mortalidade infantil e Índice de Desenvolvimento Humano por região.

Os maiores valores do índice de envelhecimento populacional foram os apresentados pelas UF que compõem as regiões Sul, Sudeste e Centro-Oeste O valor máximo foi de 29,2 no Rio de Janeiro o e mínimo, de 6,74 em Roraima, sendo o valor nacional de 19,4.

A correlação do IEP com os coeficientes de mortalidade estudados foi positiva, sendo os maiores valores de $47,9 \%$ com o $\mathrm{CMC}$ e $42,5 \%$ com o $\mathrm{CMN}$, indicando que populações com maior número de idosos apresentam maiores coeficientes de mortalidade por processos crônicos.
A Tabela 2 mostra a mortalidade proporcional por causas mal definidas (MCMD) nas UF em 2005. Os estados que compõem as regiões Norte e Nordeste apresentam os maiores valores de MCMD do país, ao passo que os que compõem as regiões Sul e Sudeste apresentam valores mais baixos. O Mato Grosso do Sul apresentou a menor MCMD, de 1,83 , e a Bahia, o maior valor de MCMD, com 25,3 . 
Tabela 2 - Mortalidade proporcional por causas mal definidas no Brasil e nas UF em 2005.

\begin{tabular}{|c|c|c|c|}
\hline UF & Óbitos & Total de óbitos & MCP \\
\hline Brasil & 104.455 & 1.006 .827 & 10,4 \\
\hline Bahia & 16.383 & 64.687 & 25,3 \\
\hline Pará & 5.924 & 26.402 & 22,4 \\
\hline Piauí & 3.149 & 14.253 & 22,1 \\
\hline Amazonas & 2.366 & 11.132 & 21,3 \\
\hline Ceará & 7.624 & 39.867 & 19,1 \\
\hline Maranhão & 3.978 & 23.931 & 16,6 \\
\hline Paraíba & 3.162 & 20.289 & 15,6 \\
\hline Amapá & 253 & 1.765 & 14,3 \\
\hline Alagoas & 2.063 & 15.311 & 13,5 \\
\hline Minas Gerais & 12.255 & 106.275 & 11,5 \\
\hline Pernambuco & 5.250 & 52.247 & 10,0 \\
\hline Sergipe & 926 & 9.787 & 9,5 \\
\hline Rondônia & 590 & 6.252 & 9,4 \\
\hline Rio de Janeiro & 10.679 & 114.394 & 9,3 \\
\hline Santa Catarina & 2.601 & 29.915 & 8,7 \\
\hline Rio Grande do Norte & 1.208 & 14.172 & 8,5 \\
\hline Acre & 217 & 2.811 & 7,7 \\
\hline Goiás & 1.923 & 26.834 & 7,2 \\
\hline Mato Grosso & 826 & 12.936 & 6,7 \\
\hline São Paulo & 14.800 & 236.456 & 6,3 \\
\hline Roraima & 89 & 1.440 & 6,2 \\
\hline Tocantins & 276 & 5.055 & 5,5 \\
\hline Rio Grande do Sul & 3.823 & 71.229 & 5,4 \\
\hline Paraná & 2.822 & 58.778 & 4,8 \\
\hline Espírito Santo & 818 & 18.676 & 4,4 \\
\hline Distrito Federal & 221 & 9.425 & 2,3 \\
\hline Mato Grosso do Sul & 229 & 12.508 & 1,8 \\
\hline
\end{tabular}

É possível observar na Tabela 2 que o estado do Maranhão, que apresenta o menor valor de CMN e de CMR, apresenta mortalidade proporcional por causas mal definidas de 16,6 para cada cem óbitos, ao passo que o Rio Grande do Sul, que apresenta o maior valor de CMN e de CMR, possui MCMD de 5,4 para cada cem óbitos. O Amapá, que possui menor valor de CMC, apresenta MCMD de 14,3 para cada cem óbitos, enquanto o Para- ná, que possui o maior CMC, apresenta MPC de 4,8 para cada cem óbitos. Essas diferenças nos MCMD evidenciam uma diferença significativa na qualidade dos dados de mortalidade nas UF.

\section{Discussão dos resultados}

Fica evidente que a transição demográfica rumo ao envelhecimento ocorre de forma heterogênea no país, estando 
esse processo mais avançado nas unidades federadas mais desenvolvidas socioeconomicamente, principalmente as que compõem as regiões Sul e Sudeste. Essa relação entre envelhecimento populacional e condição socioeconômica já foi evidenciada em estudos anteriores, como o de Kalache, Veras e Ramos (1987), que relacionaram o envelhecimento populacional de diferentes países com o seu desenvolvimento, evidenciando a correlação positiva entre essas variáveis. Chaimowicz (1997) apontou diferenças regionais no envelhecimento populacional no Brasil, relacionando-as com o grau de desenvolvimento de cada região.

Percebe-se também que a mortalidade por doenças crônicas se comporta de forma variada nas UF, acompanhando a tendência do envelhecimento populacional do país, sendo maior a mortalidade nas unidades federadas que se encontram em um estágio mais avançado de envelhecimento etário e com melhores indicadores socioeconômicos. Chaimowicz (1997), Cordeiro (1999), Lima-Costa et al. (2002) e Barros et al. (2006) destacam em seus estudos a relação entre a mortalidade e a situação socioeconômica, convergindo com os resultados encontrados no presente estudo.

Ainda quanto à mortalidade por doenças crônicas, os estados que compõem as regiões Norte e Nordeste apresentam os menores valores, o que pode estar relacionado a fatores como:

a) deficiência no atendimento à saúde, que não consegue tratar essas doenças por longos anos, não permitindo que os doentes crônicos atinjam a terceira idade; b) maior mortalidade por processos tipicamente agudos, como doenças infecciosas e parasitárias;

c) baixa qualidade do diagnóstico, não detectando a doença crônica;

d) qualidade dos dados de mortalidade.

\section{Conclusão}

As diferenças encontradas na mortalidade por doenças crônicas nas UF representam uma preocupação para o setor saúde, principalmente em relação à organização das ações e aos investimentos específicos. A compreensão da desigualdade nos processos de envelhecimento e mortalidade, bem como as mudanças que tal processo proporciona a uma população, pode ser um facilitador para intervenções diretas nos serviços com maior demanda no momento, bem como a promoção de ações que possam prevenir os problemas predominantes e pertinentes a essa transição demográfica.

Com base nos resultados encontrados, pode-se observar a necessidade de uma abordagem diferenciada para o controle das doenças crônicas no Brasil. A prevenção e o tratamento dos processos agudos, objetivando a redução dos casos crônicos, a adoção de estratégias diferenciadas, como educação em saúde e ações regionais específicas com base nos dados epidemiológicos de cada UF, e a promoção de melhorias na assistência aos casos crônicos já existentes podem gerar tratamento e controle mais eficientes, diminuindo a morbidade e a mortalidade por essas doenças, possibilitando uma melhor qualidade de vida da população idosa. 
Inequalities in mortality by chronic diseases among elderly and its association with socio-economic indicators in Brazil

\section{Abstract}

The population aging in Brazil is resulting from the fall in the taxes of birth rate and infant mortality and of the increase in life expectation. This process brings changes in the mortality of the population, when the chronic diseases are prevailing at the place of the sharp diseases. These chronic diseases produce long period of recurrent use of the health services. This change brings problems for the health system of the country that is not prepared to attend this old population in growth and to treat appropriately his chronic diseases. The understanding of the inequalities in the mortality for chronic diseases in old and his association with socio-economic indicators of Brazil can help in elderly health promotion. In this sense, statistical analysis was carried out epidemiologic, population and socioeconomics data with the finality of analyzing the inequalities in the distribution of same and to contribute in the discussion of efficient actions in the maintenance and promotion of the health of the old population. It was realized that the northern and northeast regions presented less values of the indicators socio-economics and also of the taxes of mortality for chronic diseases. These regions are in an initial stage or intermediary of population aging. The UF that make part of the south, south-east and western-centre regions are in a stage more advanced of aging, presenting elevated values of the socio-economic indicators and also of the taxes of mortality for chronic diseases. It is realized by these results that there is the necessity of an approach differentiated for the control of the chronic diseases in the Brazil and for the elderly health promotion.

Key words: Aging. Inequality. Chronic diseases. Elderly health.

\section{Referências}

BARROS, M. B. A. et al. Desigualdades sociais na prevalência de doenças crônicas no Brasil, PNAD-2003. Ciência \& Saúde Coletiva, Manguinhos, v. 11, n. 4, p. 911-926, 2006.

CHAIMOWICZ, F. A saúde dos idosos brasileiros às vésperas do século XXI: problemas, projeções e alternativas. Revista de Saúde Pública, São Paulo, v. 31, n. 2, p. 184-200, 1997.

CHIESA, A. M.; WESTPHAL, M. F.; AKERMAN, M. Doenças respiratórias agudas: um estudo das desigualdades em saúde. Cadernos de Saúde Pública, Rio de Janeiro, v. 24, n. 1, p. 55-69, jan. 2008.

CORDEIRO, R. et al. Desigualdade dos indicadores de mortalidade no Sudeste do Brasil. Revista de Saúde Pública, São Paulo, v. 33, n. 6, p. 593-601, 1999. Disponível em: <www. fsp.usp.br/rsp>. Acesso em: 18 jul. 2008.

DUARTE, E. C. et al. Expectativa de vida ao nascer e mortalidade no Brasil em 1999: análise exploratória dos diferenciais regionais. Revista Pan-Americana de Saúde Pública, v. 12 , n. 6 , p. 436-444, 1999.

ISHITANI, L. H. et al. Desigualdade social e mortalidade precoce por doenças cardiovasculares no Brasil. Revista de Saúde Pública, São Paulo, v.40, n. 4, p. 684-691, 2006.

JARDIM, V. F. S.; MEDEIROS, B. F.; BRITO, A. M. Um olhar sobre o processo do envelhecimento: a percepção de idosos sobre a velhice. Revista Brasileira de Geriatria e Gerontologia, Rio de Janeiro, v. 9, n. 2, p. 25-34, 2006.

KALACHE, A.; VERAS, R. P.; RAMOS, L. R. O envelhecimento da população mundial. Um desafio novo. Revista de Saúde Pública, São Paulo, v. 21, n. 3, p. 200-210, 1987.

LIMA-COSTA, M. F. F. et al. Diagnóstico da situação de saúde da população idosa brasileira: um estudo da mortalidade e das internações hospitalares públicas. Informe Epidemiológico do SUS 2000, v. 9, n. 1, p. 23-41, 2000. 
TRENTINI, M. et al. Enfrentamento de situações adversas e favoráveis por pessoas idosas em condições crônicas de saúde. Revista Latino-Americana de Enfermagem, São Paulo, v. 13, n. 1, p. 38-45, jan./fev. 2005. 